\title{
Paradoxical experiences of Ghanaian adolescents with HIV: physiological challenges
}

\author{
Abdul-Razak Doat, Elham Navab, Akram S. Sadat Hoseini \\ Tehran University of Medical Sciences, Iran
}

\begin{abstract}
Introduction: Adolescents living with human immunodeficiency virus (HIV) in sub-Saharan Africa continue to experience extreme health vulnerabilities. Children with HIV may experience major physiological problems. This study explored the experience of Ghanaian adolescents with HIV.

Material and methods: Semi-structured interviews were used to collect data. 12 adolescents living with HIV between the ages of 14 to 19 years were recruited. Since a physiological problem is not without some level of emotional consequence, the most appropriate method to explore these physiological and emotional dimensions of treatment and diseases are through a qualitative research method.

Results: Two main themes emerged from this study: 'Rhapsody of physiological burden' and 'Bittersweet experience of adjusting to antiretroviral therapy (ART)'. Participants reported experiencing challenges, including bouts of headaches, itchy skin rashes, general body weakness, vomiting, appetite loss, diarrhea, and many more. Adolescents reported varying accounts concerning ART. Some patients described positive image of treatment, while others reported traumatic ordeal regarding side effects of the treatment to the extent that some stopped taking their medications.

Conclusions: Policies aimed at supporting adolescents living with HIV needs to consider their unique set of physiological challenges to help improve health outcomes of the youngsters. Nurses should inform patients about results as well as possible side effects of ART prior initiation of the regimen, and also explain adverse events, which should be reported to hospital.
\end{abstract}

HIV AIDS Rev 2021; 20, 1: 59-64 DOI: https://doi.org/10.5114/hivar.2021.105087

Key words: human immunodeficiency virus (HIV), adolescent, phenomenology, lived experience, Ghana.

\section{Introduction}

Globally, at the end of 2018, it was estimated that 37.9 million people were living with human immunodeficiency virus (HIV), out of which about 1.7 million were new cases of HIV infection and 1.7 million were children below the age of 15 [1] Over $60 \%$ of HIV-positive adolescents reside in sub-Saharan Africa [2]. Youngsters living with HIV in sub-Saharan Afri-

ca continue to experience extreme health vulnerabilities [3]. The pathogenesis of HIV adolescent involves varying elements, including physical, biological, and psychological exposures; therefore, understanding of the developmental pattern of this pathogenesis is of utmost importance [4]. Children with HIV are facing challenges of treatment failure and drug resistance, which may have a long-term effect of treatment and lead to significant comorbidities [5]. HIV weakens human immune

Article history:

Received: 10.08 .2020

Received in revised form: 31.08 .2020

Accepted: 07.09.2020

Available online: 30.03 .2021
International Journal of HIV-Related Problems

HIV \& AIDS

R e vi e w 
system and affects individual's defense system against opportunistic infections. Children, who are infected with HIV at birth, may experience major metabolic complications, such as dyslipidemia and insulin resistance, problems associated with both HIV infection and several antiretroviral agents, and growing with age risk of cardiovascular disease [5]. As of 2017, 310,000 Ghanaians were infected with HIV, of which 28,000 were children between 0 and 14 years [6]. Persons infected with HIV are reluctant to report to the hospital on time, because of fear of experiencing discrimination and stigma from their community. This leads to treatment delay and progression of the disease, and eventually, resulting in acquired immunodeficiency syndrome (AIDS) stage, with the ability to develop an opportunistic infection [7]. With the inception of antiretroviral therapy (ART), $\mathrm{HIV}$, which has been previously classified as a deadly disease, has now changed into a manageable chronic condition [8]. Even though ART has brought a lot of relieve to persons living with HIV, it has also created some sort of challenges concerning its side effects in lives of people who depend on these therapies. A study by Nagpal et al. revealed that $90.6 \%$ of patients on highly active antiretroviral therapy (HAART) developed an adverse drug reaction, with 618 episodes in various systems, with the abdominal and central nervous systems being the most affected [9]. Antiretroviral therapy has proven to be very effective, being able stop HIV pathogenic effects in children living with the disease, but its toxicity is associated with neurological developmental delays experienced by HIV-positive children [10]. Moreover, studies show that antiretroviral therapy affects bone mineral density of some children younger than 12 years [11]. For some HIV patients, side effects of ART are unbearable and after a chain of events, they discontinue the treatment [12]. Children infected with HIV at birth on antiretroviral therapy tend to exhibit lower growth parameters than uninfected children, and would maintain lower level of parameters during the adolescent stage [13]. In Ghana, 24,806 adolescents are living with HIV; a great number of this population have battled years of medication use, ill health, parents' death, and physical and psychological difficulties, which are secondary to HIV and medications [14]. Adolescents' HIV-related challenges are constant; therefore it is necessary to identify these challenges and investigate them further [15]. A strong proof is needed for creative and intended solutions, which would connect research gaps, inform policy, and improve youngsters' treatment outcomes [16]. Presently, there is insufficient data on challenges of HIV-positive adolescents in Ghana; thus, the aim of this present study was to deliver freshly-gathered information on the challenges of adolescents living with HIV (ADLHIV) regarding the virus and treatment as well as benefits they derive from antiretroviral therapy. Physiological problems triggers some level of emotional challenges; therefore, the most appropriate design to explore these physiological and emotional dimensions of treatment and disease is to use a qualitative research method. Emotions dictate thinking and mobilize to action; just us feelings direct us to what we want, need, or determine our actions; they facilitate the formation of relationships and influence our overall judgment and behavior [17]. It is therefore important for nurses to be well-informed about the emotional effect of HIV and its treatment. To this end, the current study aimed to explore the experiences of adolescents living with HIV and what it means to live with the virus in Ghana.

\section{Material and methods}

This was a qualitative study; information was taken directly from study participants by first author, and were audiorecorded through semi-structured interviews with adolescents living with HIV. The study aimed to gain insight into the physiological and emotional experiences of adolescents living with the disease, and gain an understanding of real meaning of the experience of living with the disease.

\section{Study setting}

The study was conducted in Northern part of Ghana at the HIV Clinic of Tamale Teaching Hospital (TTH). The hospital is situated in the eastern part of Tamale. The facility serves as a referral hospital for people within the Northern region, Upper East, Upper West, Savana, North-East, and Bono East regions of Ghana. The clinic offers specialized care to persons living with HIV/ AIDS. The patients' population for adults is about 2,000, and that of pediatric patients is about 200 (retrieved from the records of HIV Clinic of Tamale Teaching Hospital, 2019).

\section{Study population and sampling strategy}

The inclusion criteria was to select participants living with HIV between ages 10 to 19 years, but a greater number of participants below age of 14 who visited the facility for HIV treatment with their parents/caregivers did not know their HIV status, though they were on ART. For this reason, the research team was unable to include them in the study, thus we had to rely on adolescent who were aware of their HIV status. ADLHIV between the ages of 14 and 19 years were recruited for the study, with average of 16.9 years. The participants were recruited from a HIV clinic of tertiary facility, which serves as a referral hospital for the Northern sector of Ghana. Eight females and four males living with HIV were recruited for this study. A purposive sampling technique was used to recruit as well as to interview the participants. Adolescent living with the disease were selected for the study if they met the following inclusion criteria: adolescents living with HIV in the Northern sector of Ghana, mentally sound and stable, age bracket of 10-19 years, known HIV status, and willingness to take part in the study.

\section{Data collection}

From November 2019 till March 2020, data were collected by the first author through one-on-one in-depth interviews, which were audio-taped and transcribed verbatim immediately after each interview. Each interview lasted 
between 30 and 60 minutes, and participants could choose the time and venue for the interview. The choice of venue was dependent on where the participant felt safe. ADLHIV were asked to share their experience of living with HIV. Participants were asked to respond to an open-ended question: "What is the meaning of living as an adolescent with HIV in Ghana?". They were encouraged to freely express themselves about their experiences, and follow-up questions were asked for clarifications whenever needed. Data collection and analysis were concurrent and continued till a deep, rich, related, and abstract themes were completed.

\section{Statistical analysis}

Data analysis was performed using Van Manen's six activities; it started from the third activity of Van Manen, where themes that characterized the essential phenomenon were reflected on. During this stage, audio-recorded interviews were transcribed into plain text and verbatim immediately after each interview. Researchers listened to audio and read the transcripts severely to avoid errors. Thematic analysis was done with gathered data in other to obtain the essential meaning of an experience. A holistic and selective approach was employed for the exploration of themes. Each transcript was read and a brief-written. In a selective approach, the individual transcript was read line by line, and statements, which reflected the research question were identified. In the fourth step, the adolescent phenomenon was described through writing and rewriting in other to obtain a very good description of the phenomenon. Research team indulged in writing till we obtained an essential meaning of the phenomenon. The fifth step involved staying on track with the research question. The team maintained a strong and oriented relationship with the phenomenon by trying to understand the lived experiences of ADLHIV from their perspective. We recorded our preconceived opinion, conceptions memories, and experiences in a journal/diary throughout the study. We reflected, spoke, and wrote in a manner that is both oriented and strong in a pedagogic sense. In the sixth and final step, we balanced the research context by checking the parts and the whole, and referred to the analysis of individual interviews and the entire stock of data, looking for keywords, concepts, sub-themes, and themes.

\section{Ethics}

Approval of this study was obtained from the ethics committee of Tehran University of Medical Sciences (code: IR. TUMS.FNM.REC.1398.091). The research unit of the hospital granted permission for the study to be conducted on their premises. A written consent was obtained from each participant after explaining the purpose of the study. For participants who were below the age of consent, a written consent and permission to record was taken from their parents/caregivers. Anonymity and confidentiality of participants were maintained by not recording patients' names and addresses.

\section{Trustworthiness}

In this study, researchers ensured credibility by constantly engaging with data during all stages of the study and performing collaborative analysis. This approach minimized the consequence of individual researcher's predetermined notion on the study outcome. All the steps of this study was scrutinized with supervisors and panel of experts; their recommendations were factored into the study to ensure accuracy and appropriateness. It is important to note that member checking process was also employed to validate interpretations and classification.

\section{Results}

The age range of participants recruited for this study was between 14 and 19 years, with an average age of 16.91 years. $50 \%$ of participants were between 18 and 19 years old, while the remaining $50 \%$ were between 14 and 16 years of age. $50 \%$ of participants were junior secondary school graduates, $25 \%$ were senior secondary school graduates, while the remaining $25 \%$ had never been to school. $42 \%$ of participants were coming from the most dominant tribe in the Northern region of Ghana, i.e., Dagomba tribe. The remaining 58\% was shared amongst participants who came from other parts of Ghana, such as Savanna, Volta, Upper-East, Bono-East, and Northeastern regions of Ghana. The participants recruited for this study were all HIV-positive and on antiretroviral therapy. All participants were receiving treatment at the HIV Clinic of Tamale Teaching Hospital. Two main themes emerged from this study: 'Rhapsody of physiological burden', and 'Bittersweet experience of adjusting to ART'.

\section{Rhapsody of physiological burden}

Some ADLHIV reported that they were often falling sick and going to the hospital with signs and symptoms that were not subsiding, and even if they subsided for a short while, they would recur even more intense. They tried several treatments only to realize it was HIV, and this was after a series of laboratory tests performed. Some of them also indicated that they were regularly falling sick and were constantly on some medication, but they really did not know they were living with HIV, until it was disclosed to them by their parents or guardians. Two sub-themes emerged from this study, including 'Agony of living with body discomfort' and 'Disintegration of vital physical stature'.

\section{Agony of living with body discomfort}

Participants of the study reported that they had lots of challenges in the early days of the disease. These challenges ranged from bouts of headache, severe abdominal pain, itchy skin rashes, general body weakness, vomiting, appetite loss, diarrhea, and could not perform some activities of daily living because of running out of breath. 
"What I went through, it was not easy. I was sick, I could not eat. I did not know what was wrong with me till I found out, so I really suffered. Even if there is food, I cannot eat. I will be feeling hungry, but I can't eat and there is nothing that is appetizing to me" (Participant No. 4).

"I was having stomach pain, headache, and even running diarrhea. The way it pained me hmmm, the stomach pain was so severe and when it starts, I cannot even straighten myself, I will just be lying down. As for the headache, the headache will prick all your head and you cannot even see or you cannot even wake up, you will be lying down just like that" (Participant No. 11).

\section{Disintegration of physical stature}

Some ADLHIV recounted that in the course of the disease, they grew lean and were no longer looking attractive. They experienced rapid weight loss and the only visible features were just the boney prominence; some suffered hair loss, skin peeling, and skin rashes.

"I had lost everything, even lost my hair, I didn't have buttocks again, when I sit for long, I feel pain around my buttocks because I am now sitting on bones and there was no meat around me again" (Participant No. 12).

"I completely run down, I completely lost weight, the second was that my skin was peeling out, and you could see my body as a skeleton. Another thing I saw in my body was that my hair was coming off, I was no longer having hair" (Participant No. 10).

\section{Bittersweet experience of adjusting to antiretroviral therapy}

ADLHIV shared varying accounts of taking antiretroviral therapy. Some described experiencing enormous relief as a result of commencing antiretroviral therapy, they indicated that the positive effect of the drugs brought to them a long-awaited comfort they had longed for. They also expressed satisfaction to the effect that the antiretroviral treatment has improved their lives and boosted their image positively. While a group of respondents were full of praise on the positive effects of the antiretroviral therapy, others gave a harrowing account of the ordeal they had to endure when started highly active antiretroviral therapy. It was to the extent that some even stopped taking their medications. Two sub-themes emerged from this study, such as 'The magic of antiretroviral therapy (ART)' and 'Burden of antiretroviral therapy (ART)'.

\section{The magic of antiretroviral therapy}

All participants who were enrolled in the study praised the positive impact of ART on their lives. ADLHIVs who previously could not perform activities of daily living because of breathlessness, weakness, and some other challenges, were now able to perform activities unaided, and were encouraged to pursue their future ambitions. Most of the participants reported that they no longer experience headaches, diarrhea, appetite loss, and all the other manifestations they use to encounter before they started ART.

"ART has made me fine, it has made me stronger, fit, and healthy, and it has improved my appetite. I am always active" (Participant No. 1).

"The ART medication has kept me alive and that is what is keeping me going. I have gained a lot from the medicine because this is what has kept me strong, it has helped me to eat" (Participant No. 7).

"As for the antiretroviral therapy is the best treatment for HIV diagnosis because when I started taking it, I got results, I could see that all this while I was going around looking for herbal drugs, which were not working, I was wasting my time" (Participant No. 12).

"As for antiretroviral therapy, nobody will tell me that it is not drugs for HIV. I was weak, now I am strong, when my body was just looking pale, I have now recovered, my jaw was just something else, I can now eat, I can now walk, I can do anything for myself, and I can even do more than an HIV-negative person" (Participant No. 12).

\section{The burden of antiretroviral therapy}

Although ART has brought so much relief to persons living with HIV, ADLHIV shared their experiences concerning the adverse drug reactions they encountered. Participants' experiences ranged from excruciating headaches, diarrhea, nausea and vomiting, strange dreams, sleeplessness, abdominal pains, body weakness, seizures, aggressiveness, and skin rashes.

"Most times when I take the drugs, I become aggressive. When you are with me, I will do to you, you would not like it, but I will sit down and regret my actions, but is not my intention to be aggressive to people" (Participant No. 1).

"When I was diagnosed HIV-positive and they put me on antiretroviral therapy (ART), I had so many side effects of the drugs; it was headache, nausea, itching of the skin, and also skin rashes, and the worst thing was diarrhea and sometimes vomiting. As time went by, when I started taking the drugs and the side effects continued, I stopped taking the drugs" (Participant No. 10).

"When I started taking the HIV drugs, I experienced headaches and some rashes, and I was even having bad dreams of something chasing me in my dreams or someone is going to kill me" (Participant No. 11).

\section{Discussion}

In this study, 'Rhapsody of physiological burden' and 'Bittersweet experience of adjusting to ART' emerged as the major themes, with its accompanying sub-themes. For 'Rhapsody of physiological burden', the sub-themes were 'Agony of living with body discomfort' and 'Disintegration of vital physical stature'. The sub-themes that emerged from 
'Bittersweet experience of adjusting to ART' included 'Magic of ART' and 'Burden of ART'. ADLHIV recounted the level of body discomfort they had to bear as a result of the unbearable nature of HIV symptoms. These symptoms made it difficult to perform various activities of daily living. The majority of participants reported experiencing headaches, abdominal pains, appetite loss, body weakness, nausea, and vomiting in addition to a host of other symptoms. Their account is corroborated by the findings of a study performed on people living with HIV in Eastern Cape, South Africa, which revealed that headaches, fever, fatigue, weakness, painful joints, nausea, muscle aches, and dizziness were among the top 10 symptoms manifested by participants [18]. In a similar study investigating the difference in clinical manifestations of acute and early HIV infection in African women, the results showed that unexplained fever, weight loss, fatigue, and headaches were amongst the symptoms manifested by participants [19]. In another study, loss of appetite and headache were amongst the most prominent signs and symptoms reported by people living with HIV [20].

Some ADLHIV also described experiencing vivid wasting and losing weight in addition to losing hair in the course of the disease. Weight loss in patients infected with HIV is associated with disease progression and death [21]. In another study, weight loss was found to start early in the disease, before any significant compromise in immune status [22]. Studies also show that the presence of apoptotic or necrotic keratinocytes within the upper end of the external root sheath epithelium and dystrophy of hairs, may be markers of hair loss in patients with HIV-1 infection [23].

ART medication has brought a great sigh of relief to ADLHIV; the participants reported the joy of waking up from bed without noticing key symptoms that kept them down and helpless. The participants stated that when they were on ART, the constant, unending signs and symptoms they used to have were no longer there. They recovered from all the key symptoms, including wasting and loss of appetite, and this has helped them regain their self-image. This is validated by findings from a study investigating the benefits of ART in patients living with HIV; the study revealed that probability of experiencing pain in a previous week decreased from $69 \%$ in months preceding ART initiation to $17 \%$ after 5 years ART, and fatigue from $62 \%$ to $7 \%$. The study further revealed that the probability of not being able to perform normal activities in a previous week fell from $47 \%$ to $5 \%$. Also, being employed increased from $32 \%$ to $44 \%$ and difficulty with job performance among those employed fell from $56 \%$ to $6 \%$. As health improved, the probability of relying on a caretaker declined from $81 \%$ to less than $1 \%$, and receipt of a disability grant, which initially increased, fell slightly overtime during ART [24]. Initiating ART is associated with reduced mortality compared with delaying such therapy [25]. Noteworthy, findings from a study conducted to assess survival benefits of antiretroviral therapy in South Africa showed that an estimated population lifetime survival benefit for all people starting ART during 2004-2011 was
21.7 million life years, of which 2.8 million life years (12.7\%) had been realized by December 2012 [26].

A group of ADLHIV indicated that, when they were put on ART, they encountered some side effects, such as dizziness, nausea, and vomiting, headaches, body weakness, pains in the legs and arms, insomnia, strange dreams, itching of the skin, skin rashes, diarrhea, aggressive, and seizures. This is supported by other studies, where the habitual adverse effect of some antiretroviral treatment, such as zidovudine (AZT) are fatigue and headache, whereas efavirenz (EFV) was found to cause nightmares [27]. Other studies revealed that regular, but mild adverse effects that appear early in most antiretroviral regimens, include gastrointestinal effects, such as bloating, nausea, and diarrhea, which may be transient or may persist throughout therapy [28]. In a related study to assess the impact of ART side effects on quality of life of Chinese HIV-positive individuals, the findings revealed that participants experienced digestive discomforts, skin rashes, numbness, memory losses, nightmares, and dizziness [29].

\section{Conclusions}

The present study described some of the challenges and adverse effects, which adolescents living with HIV encountered prior to starting of ART and while on ART. The study also described the positive impact of ART in lives of participants, who initially had difficulties performing activities of daily living. ADLHIV could proceed with their normal daily activities because of the positive impact of ART. The physiological burden encountered by ADLHIV included bouts of headaches, severe abdominal pains, itchy skin rashes, general body weakness, vomiting, appetite loss, and diarrhea. The adverse effects of ART manifested by participants were excruciating headaches, diarrhea, nausea and vomiting, strange dreams, sleeplessness, abdominal pains, body weakness, seizures, aggressiveness, and skin rashes. Policies aimed at supporting ADLHIV need to consider these physiological challenges to help optimize health outcomes for adolescents. Nurses should inform ADLHIV about the effects and possible side effects of ART prior to starting the regimen, and also explain adverse effects, which should be reported to the hospital as well as adverse effects that demand the continuation of ART. Nursing care plan for adolescents living with HIV must incorporate measures that will help promote comfort and bring relief to those youngsters who manifest any of the signs and symptoms prior to taking ART or while on ART. Nurses should fashion out health education and interventions on HIV and ART, which is easily assessable to adolescents, and this intervention should be tailored to meet the needs of ADLHIV in their communities. Nurses could use the advancement in technology, such as social media platforms, radio, and television to accomplish this task. The study was performed on a limited number of respondents, which did not permit the study to be generalized. 


\section{Conflict of interest}

The authors declare no conflict of interest with respect to the research, authorship, and/or publication of this article.

\section{References}

1. Mahy M, Marsh K, Sabin K, Wanyeki I, Daher J, Ghys PD. HIV estimates through 2018: data for decision-making. AIDS 2019; 33 (Suppl 3): S203-S211.

2. UNICEF. For every child, end AIDS: seventh stocktaking report, 2016. UNICEF; 2016.

3. Cluver L, Pantelic M, Orkin M, Toska E, Medley S, Sherr L. Sustainable Survival for adolescents living with HIV: do SDG-aligned provisions reduce potential mortality risk? J Int AIDS Soc 2018; 21 Suppl 1: e25056.

4. Naswa S, Marfatia Y. Adolescent HIV/AIDS: issues and challenges. Indian J Sex Transm Dis 2010; 31: 1-10.

5. Vreeman RC, Scanlon ML, McHenry MS, Nyandiko WM. The physical and psychological effects of HIV infection and its treatment on perinatally HIV-infected children. J Int AIDS Soc 2015; 18 (Suppl 6): 20258.

6. Ghana AIDS Commission. Summary of the 2016 HIV sentinel survey report, 2017; 2018.

7. Balatif R. HIV infection: what should we know? Journal of Endocrinology, Tropical Medicine, and Infectiouse Disease (JETROMI) 2020; 2: 1-16.

8. Posse M, Baltussen R. Barriers to access to antiretroviral treatment in Mozambique, as perceived by patients and health workers in urban and rural settings. AIDS Patient Care STDs 2009; 23: 867-875.

9. Nagpal M, Tayal V, Kumar S, Gupta U. Adverse drug reactions to antiretroviral therapy in AIDS patients at a tertiary care hospital in India: a prospective observational study. Indian J Med Sci 2010; 64 : 245-252.

10. Willen EJ. Neurocognitive outcomes in pediatric HIV. Ment Retard Dev Disabil Res Rev 2006; 12: 223-228.

11. Gafni RI, Hazra R, Reynolds JC, et al. Tenofovir disoproxil fumarate and an optimized background regimen of antiretroviral agents as salvage therapy: impact on bone mineral density in HIV-infected children. Pediatrics 2006; 118: e711-e718.

12. Shabalala FS, Vernooij E, Pell C, et al. Understanding reasons for discontinued antiretroviral treatment among clients in test and treat: a qualitative study in Swaziland. J Int AIDS Soc 2018; 21 Suppl 4: e25120.

13. Buonora S, Nogueira S, Pone MV, Aloé M, Oliveira RH, Hofer C. Growth parameters in HIV-vertically-infected adolescents on antiretroviral therapy in Rio de Janeiro, Brazil. Ann Trop Paediatr 2008; 28: 59-64.

14. Enimil A, Nugent N, Amoah C, et al. Quality of life among Ghanaian adolescents living with perinatally acquired HIV: a mixed methods study. AIDS Care 2016; 28: 460-464.

15. Ayres JRdCM, Segurado AAC, Galano E, et al. Adolescentes e jovens vivendo com HIV/aids: cuidado e promoção da saúde no cotidiano da equipe multiprofissional. Sao Paulo; 2004.

16. Armstrong A, Nagata JM, Vicari M, et al. A global research agenda for adolescents living with HIV. J Acquir Immune Defic Syndr 2018; 78 Suppl 1: S16-S21.

17. Rice LN, Elliott R. Facilitating Emotional Change: The Moment-byMoment Process. Guilford Press; 1996.

18. Peltzer K, Phaswana-Mafuya N. The symptom experience of people living with HIV and AIDS in the Eastern Cape, South Africa. BMC Health Serv Res 2008; 8: 271.

19. Lemonovich TL, Watkins RR, Morrison CS, et al. Differences in clinical manifestations of acute and early HIV-1 infection between HIV-1 subtypes in African women. J Int Assoc Provid AIDS Care 2015; 14: 415-422.
20. Mlisana K, Sobieszczyk M, Werner L, et al. Challenges of diagnosing acute HIV-1 subtype C infection in African women: performance of a clinical algorithm and the need for point-of-care nucleicacid based testing. PLoS One 2013; 8: e62928.

21. Tang AM, Forrester J, Spiegelman D, Knox TA, Tchetgen E, Gorbach SL. Weight loss and survival in HIV-positive patients in the era of highly active antiretroviral therapy. J Acquir Immune Defic Syndr 2002; 31: 230-236.

22. Mangili A, Murman D, Zampini A, Wanke C, Mayer KH. Nutrition and HIV infection: review of weight loss and wasting in the era of highly active antiretroviral therapy from the nutrition for healthy living cohort. Clin Infect Dis 2006; 42: 836-842.

23. Smith KJ, Skelton HG, DeRusso D, et al. Clinical and histopathologic features of hair loss in patients with HIV-1 infection. J Am Acad Dermatol 1996; 34: 63-68.

24. Rosen S, Larson B, Rohr J, et al. Effect of antiretroviral therapy on patients' economic well being: five-year follow-up. AIDS 2014; 28 : 417-424.

25. Palella FJ, Deloria-Knoll M, Chmiel JS, et al. Survival benefit of initiating antiretroviral therapy in $\mathrm{HIV}$-infected persons in different CD4+ cell strata. Ann Intern Med 2003; 138: 620-626.

26. April MD, Wood R, Berkowitz BK, et al. The survival benefits of antiretroviral therapy in South Africa. J Infect Dis 2014; 209: 491-499.

27. Montessori V, Press N, Harris M, Akagi L, Montaner JS. Adverse effects of antiretroviral therapy for HIV infection. CMAJ 2004; 170 : 229-238.

28. Carr A, Cooper DA. Adverse effects of antiretroviral therapy. Lancet $2000 ; 356:$ 1423-1430.

29. Chen WT, Shiu CS, Yang JP, Simoni JM. Antiretroviral therapy (ART) side effect impacted on quality of life, and depressive symptomatology: a mixed-method study. J AIDS Clin Res 2013; 4: 218. 